\title{
Relación del rendimiento de una empresa, con su tamaño, uso de la tecnología y la capacitación: análisis de las pymes de servicios en un destino turístico
}

\author{
Oliver Cruz Milán* \\ Universidad de Texas-Pan American \\ Ricardo Jimeno Espadas \\ Ricardo Sonda de la Rosa \\ Universidad del Caribe
}

\section{Resumen}

Se identifica la relación que guardan el tamaño de la empresa, el uso de la tecnología, la capacitación, los años de experiencia y el tipo de servicio con el rendimiento en empresas consideradas pequeñas y medianas (pymes) en un destino turístico. Con el análisis de regresión múltiple, se examinan los efectos principales y de interacción entre estos factores. Los resultados empíricos sugieren que algunas variables independientes son predictivas del rendimiento de las pymes y que existen efectos de interacción significativos entre ellas. Al final, se discuten algunas implicaciones y conclusiones al respecto.

\section{Palabras clave}

Pymes, rendimiento, servicios, turismo. 


\title{
Relation of business size, technology use and training with performance: analyzing services SMEs in a tourism destination
}

\author{
Oliver Cruz Milán* \\ Universidad de Texas-Pan American \\ Ricardo Jimeno Espadas \\ Ricardo Sonda de la Rosa \\ Universidad del Caribe
}

\begin{abstract}
This work identifies the relation with performance of factors such as size of the firm, technology use, training, years in business, and type of service in small and medium-sized enterprises (SMEs) in a tourism destination. By employing multiple regression analysis, the main as well as interaction effects of these factors are examined. The empirical results suggest that some of the independent variables are predictors of SMEs' performance, and that significant interaction effects exist between them. Some implications are discussed and conclusions are drawn.
\end{abstract}

KeY WORDS

SMEs, performance, services, tourism.

*E-mail: ocruz4@utpa.edu •rjimeno@ucaribe.edu.mx•rsonda@ucaribe.edu.mx 


\section{Introducción}

En años recientes, tanto compañías privadas como instituciones gubernamentales y universidades de diferentes partes del mundo han reconocido la necesidad de examinar el papel y la efectividad de los diferentes tipos de intervenciones dirigidas a pequeños negocios (APEC, 2007). Su contribución a la prosperidad y solidez económica de las naciones ha sido ampliamente valorada en diversos países (Matlay, 1999). Se ha destacado que el apoyo a las pequeñas y medianas empresas (pymes) trae consigo múltiples beneficios, como una más eficiente asignación de recursos, mayor equidad en la distribución de ingresos y reducción del subempleo al ser laboralmente intensivas (You, 1995).

México es uno de los países que ha mostrado mayor interés en apoyar a las pymes, en especial a aquellas pertenecientes a la industria de viajes y turismo (Sectur, 2007). Sin embargo, no se ha realizado suficiente investigación para explicar el desempeño y rendimiento de las pymes que brindan servicios turísticos en el país (Cestur, 2007). Por lo tanto, el objetivo de este trabajo es presentar y someter a prueba un modelo que especifica la relación que guardan el tamaño de la empresa, el uso de la tecnología, la capacitación, los años de funcionamiento y el tipo de servicio con el rendimiento de la misma. El contexto de la investigación corresponde a Cancún, Quintana Roo, México, ciudad con una de las más altas tasas de formación y creación de pymes en el nivel nacional (Boggio Vázquez y Vecino Romero, 2008). En la siguiente sección se elabora el marco conceptual a partir de investigaciones sobre la influencia de varios aspectos asociados al rendimiento de estas empresas. Después se plantean las hipótesis a examinar, seguidas del diseño de la investigación y la metodología utilizada. Finalmente se exponen el análisis de los datos y la discusión de resultados.

\section{Marco conceptual}

Solo una pequeña parte de las empresas que participan en la economía y los mercados internacionales corresponde a grandes corporaciones o consorcios multinacionales, la mayoría pertenece al segmento de pymes comerciales y de servicios. En México, más de 99 \% de los negocios establecidos son clasificados como pymes, y casi todas son consideradas micro, seguidas de aquellas de 
tamaño pequeño y, por último, las de tamaño mediano (Sectur, 2007). Esta clasificación se basa en el número de empleados que poseen: son micro si tienen menos de diez; pequeñas, entre 11 y 50, y medianas, entre 51 y 250 (Secretaría de Economía, 2002). Conforme a la información generada por el Instituto Nacional de Estadística y Geografía (INEGI), las pequeñas empresas de todas las industrias y sectores representan más de dos tercios del empleo formal en todo el país y aportan aproximadamente 80 \% del producto interno bruto (Hernández et al., 2007). Esto demuestra la importancia de estudiar el funcionamiento y desarrollo de las pymes, incluidas las del sector turístico, debido a su contribución al crecimiento económico y social de las naciones (Singh, Reynolds y Muhammad, 2001; Wanhill, 2000).

De acuerdo con la teoría de recursos-ventajas (Hunt, 2010), las empresas que logran ventajas competitivas lo hacen por el despliegue de sus recursos y el aprovechamiento de ventajas comparativas, las cuales pueden llegar a ser sostenibles a través del tiempo debido a la gestión y el aprendizaje organizacional, teniendo como referencia central el desempeño financiero de la firma. Según esta teoría, los recursos son entidades tangibles e intangibles que se encuentran a disposición de la empresa y le permiten producir eficiente y efectivamente una oferta con valor para los consumidores. Por ejemplo, dentro de los diversos recursos disponibles se pueden incluir la magnitud de las operaciones, las herramientas tecnológicas o los conocimientos y habilidades del personal, que en conjunto sirven para satisfacer los segmentos de mercado de la firma. Además, ya que parte de su desempeño se debe a una adecuada gestión estratégica de sus recursos y capacidades, se puede inferir que hay una correlación entre el éxito de las empresas y el tiempo que estas tienen en funciones (Hunt y Morgan, 1995). En este sentido, la orientación al mercado es un elemento fundamental en el desarrollo de ventajas competitivas, por lo cual los servicios han cobrado especial relevancia dentro del contexto de la dinámica económica actual (Vargo y Lusch, 2004). Por lo tanto, es necesario considerar varios de estos aspectos al estudiar el rendimiento en las empresas.

Respecto al tamaño, los pequeños establecimientos comerciales suelen enfrentar mayores dificultades que los más grandes, en parte por los limitados recursos disponibles para invertir en estrategias de mercado o hacer mejoras (Van Dijk, 2000). Con base en su investigación sobre este tipo de negocios, Ra- 
ymond y St-Pierre (2010) señalan que el tamaño de la empresa es determinante entre los diversos aspectos relacionados con el desempeño de las pymes. Otros trabajos recientes también han encontrado que el tamaño influye en el crecimiento de las pymes y que las pequeñas están más limitadas que las grandes (Serrasqueiro et al., 2010). Así, se ha planteado que la eficiencia en las grandes empresas es mayor que en las pequeñas por el uso de técnicas industriales y de gran escala por parte de aquellas (Yang y Chen, 2009).

Otra variable relevante es el uso de las tecnologías de las información (ті) en las funciones del negocio. Las pymes se encuentran bajo una creciente presión para adquirir y emplear tecnologías de forma más eficaz con el fin de mantener su posición competitiva y sobrevivir en el mercado actual (Montazemi, 2006). Sin embargo, aún existen diferentes barreras para las pymes que desean adoptar y obtener ventajas de las soluciones tecnológicas (Dai, 2010). En la medida en que las empresas se desarrollan también es más probable que implementen tecnologías de manera más rápida y adecuada, a diferencia de lo que pueden hacer empresas más pequeñas. Al respecto, Ford y Brown (2010) han manifestado que la mayor parte de la investigación sobre la adopción y el uso de tecnologías se ha concentrado en las grandes corporaciones, pero no en las pymes. Por ende, se esperaría que al aumentar la intensidad en la utilización de las tecnologías, las pymes mejoren su eficiencia operativa y su competitividad estratégica (Ford y Brown, 2010).

La formación y capacitación de los empleados es otra variable que se suma a las ya mencionadas. Kitching y Blackburn (2002) definen la formación como cualquier esfuerzo dentro de la organización con el propósito de incrementar las habilidades relacionadas con el trabajo y el conocimiento del personal. Esta práctica ha sido ampliamente difundida porque se considera que, por medio de la formación de sus empleados, las pymes pueden mejorar considerablemente su productividad y rendimiento (Massey, 2004). De hecho, son evidentes los esfuerzos gubernamentales por impulsar programas y políticas públicas dirigidos a fomentar el desarrollo de las pequeñas empresas mediante la capacitación de su personal (Patton, Marlow y Hannon, 2000). No obstante, Storey y Westhead (2004) advierten que este tipo de empresas realizan menos acciones de formación de sus colaboradores, a pesar de que la capacitación juega un papel crucial en el crecimiento y en los resultados operativos y financieros de 
las pymes (Jayawarna, Macpherson y Wilson, 2007). Por ello, es de suponer que las organizaciones con mayor capacitación de personal son propensas a mostrar indicadores de desempeño más favorables en comparación con las que no la llevan a cabo.

Por otro lado, uno de los mayores desafíos que enfrentan las pymes es la intensa competencia con empresas que han estado en el mercado por más tiempo, lo cual sugiere que aquellas firmas que han mantenido operaciones a lo largo de los años han tenido algún éxito en la creación de valor para el cliente, traducido en resultados positivos (Olander, Hurmelinna-Laukkanen y Mahonen, 2009). Una forma de evaluar el éxito en las empresas es considerar el número de años que llevan en funciones (Serrasqueiro et al., 2010); las pymes con más tiempo en operación son propensas a un mejor desempeño, lo que a su vez se vincula con la mejora en algunos de sus procesos internos. De este modo, se plantea que el número de años en funcionamiento de las empresas se relaciona con su crecimiento, así como con el nivel de adopción de tecnologías en sus actividades.

Entre los diferentes tipos de servicios, los turísticos representan uno de los sectores productivos más valiosos para México. Según datos sobre su participación en las actividades económicas, las relacionadas con los viajes y el turismo contribuyen con alrededor de $9 \%$ del producto interno bruto nacional (Sectur, 2011). Cancún es un conocido destino turístico situado en la región del país que recibe la mayor cantidad de dólares y visitantes por este concepto (INEGI, 2010), con una alta concentración de pymes que ofrecen distintos tipos de servicios (SIEM, 2008). Dentro de la diversidad de establecimientos turísticos se encuentran servicios de alojamiento, restaurantes, transportes, entretenimiento y ocio, así como otros dirigidos específicamente a los viajeros y visitantes (Goeldner, Ritchie y McIntosh, 2000). Desde su fundación, hace casi cuatro décadas, Cancún se convirtió en un sitio donde los viajeros internacionales acuden a demandar una variedad de productos y servicios turísticos sofisticados y de calidad (Torres, 2002). Debido a este posicionamiento, es de suponer que las pymes relacionadas con el turismo sean más competitivas y tengan mejor desempeño que las no turísticas (Wood, 2006), ya que tienen la ventaja de ubicarse en uno de los destinos turísticos más reconocidos del mundo. Así, se plantea que el tipo de servicio brindado se vincula con otros factores clave, como el grado de 
crecimiento de la empresa, su nivel de uso de tecnologías y la incidencia en actividades de capacitación y formación de su personal.

Para medir el impacto e interacción de los factores mencionados es común utilizar el rendimiento o desempeño de las pymes. Aunque existen distintas formas de medir el rendimiento derivado del funcionamiento de la empresa, como los indicadores financieros u operativos, el volumen de ventas es considerado una medida adecuada para estudiar el caso de las pymes (Cosh, Duncan y Hughes, 1998). Además, puesto que muchas pequeñas empresas no cuentan con prácticas de contabilidad formales, se trata de información fácil de obtener o de recuperar en las empresas, las cuales además son dirigidas muchas veces por administradores no profesionales (Patton, Marlow y Hannon, 2000).

\section{Hipótesis de investigación}

Con base en las consideraciones mostradas, este trabajo plantea que las pymes con grandes operaciones, que utilizan más tecnología y que capacitan más a sus empleados tienden a un mejor rendimiento en comparación con aquellas que no cumplen con estas características. Así pues, se generaron las siguientes hipótesis:

$\mathrm{H}_{1}$ : El tamaño tiene un impacto directo y positivo en el rendimiento de las pymes

$\mathrm{H}_{2}$ : El uso de tecnologías de la información tiene un impacto directo y positivo en el rendimiento de las pymes

$\mathrm{H}_{3}$ : La capacitación del personal tiene un impacto directo y positivo en el rendimiento de las pymes

Además de la influencia directa de las variables descritas, deben considerarse algunos efectos por interacción con otras variables. Una interacción o moderación se manifiesta cuando la relación entre alguna variable independiente y la variable dependiente depende a su vez de otras variables independientes contempladas dentro del mismo modelo (Sharma, Duran y Gur-Arie, 1981).

Ante ello se espera detectar efectos de interacción en algunas variables, por ejemplo entre los años de funcionamiento, el tipo de servicio y las variables independientes con efectos directos sobre el rendimiento de la empresa. Por lo 


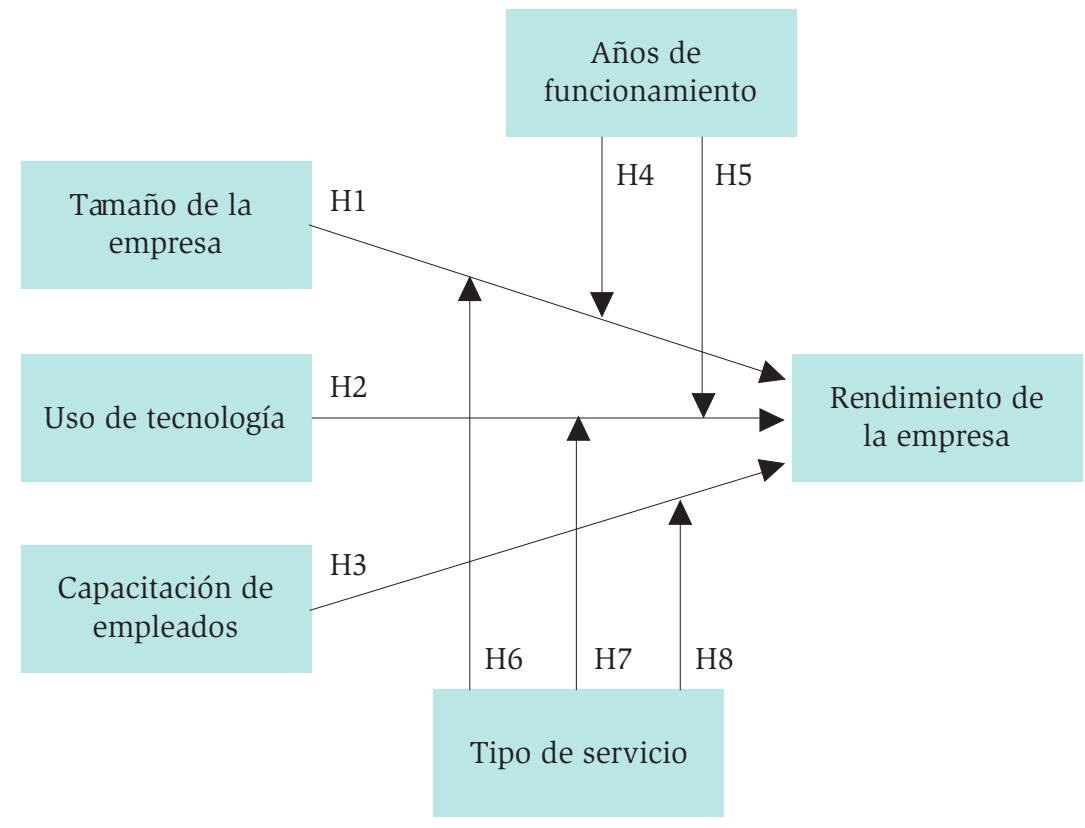

Fuente: Elaboración propia

Figura 1. Modelo con las hipótesis sobre determinantes de rendimiento en pymes

tanto, además de las hipótesis de efectos principales, se generaron las siguientes sobre estas interacciones:

H4: Hay un efecto de interacción significativo entre los años de funcionamiento y el tamaño de la empresa que impacta el rendimiento, el cual se refleja en un mayor nivel de ventas en las pymes con más años y de mayor tamaño

H5: Hay un efecto de interacción significativo entre los años de funcionamiento y el uso de tecnologías en la empresa que impacta el rendimiento, el cual se refleja en un mayor nivel de ventas en las pymes con más años y que utilizan tecnologías

H6: Hay un efecto de interacción significativo entre el tipo de servicio y el tamaño de la empresa que impacta el rendimiento, el cual se refleja en un mayor nivel de ventas en las pymes turísticas de mayor tamaño 
H7: Hay un efecto de interacción significativo entre el tipo de servicio y el uso de tecnologías en la empresa que impacta el rendimiento, el cual se refleja en un mayor nivel de ventas en las pymes turísticas que utilizan tecnologías

H8: Hay un efecto de interacción significativo entre el tipo de servicio y la capacitación en la empresa que impacta el rendimiento, el cual se refleja en un mayor nivel de ventas en las pymes turísticas que realizan capacitación

En la figura 1 se ilustran las variables independientes identificadas en el marco conceptual, así como las hipótesis de investigación. Cabe mencionar que en la literatura hay diferentes trabajos que contemplan otros factores que también podrían relacionarse con el desempeño de las pymes. Sin embargo, para fines de este proyecto solo se examinan las relaciones planteadas, ya que un análisis exhaustivo del efecto de otros constructos implicaría un objetivo y un diseño de la investigación distintos de los aquí establecidos.

\section{Metodología}

Este trabajo se deriva de la colaboración con el Observatorio de la Pequeña y Mediana Empresa de la Universidad del Caribe en Cancún, Quintana Roo, México. Por medio de una encuesta ómnibus se recopilaron datos sobre varios aspectos relacionados con las pymes de la región, los cuales sirven como base en las pruebas de hipótesis del modelo propuesto en la investigación. El cuestionario fue originalmente desarrollado y validado por el Banco Interamericano de Desarrollo (BID), y es el instrumento oficial adoptado por el gobierno federal mexicano para llevar a cabo los proyectos auspiciados en conjunto con el BID. Este instrumento se considera pertinente para el estudio ya que fue diseñado específicamente para el contexto de América Latina y se adapta a las características comunes de las pymes en México. La operacionalización de los reactivos utilizados puede ser consultada en el apéndice al final de este artículo.

Los sujetos de interés fueron las pymes de servicios en la ciudad de Cancún. Después de descartar encuestas con datos incompletos, se contó con 247 casos útiles para el análisis, de los cuales se descartaron aquellos con valores atípicos, por lo que en el análisis final se incluyeron 242 casos. Este tamaño de la muestra 
es considerado adecuado para el procedimiento de pruebas estadísticas, ya que cumple con el rango mínimo deseado de entre 15 y 20 observaciones para cada variable independiente sugerido por Hair et al. (2010).

Puesto que el propósito del trabajo es evaluar el impacto de variables predictivas sobre una variable dependiente, se decidió emplear regresiones múltiples para someter a prueba las hipótesis de investigación, pues esta técnica sirve para estudiar la magnitud de los efectos en relaciones propuestas a partir de un modelo teórico (Kerlinger y Lee, 2000; Warner, 2008). Para ello se constató que los datos cumplieran con los supuestos de homocedasticidad, linealidad, no colinealidad y normalidad. Como se describe en el cuadro 1, se cumplió con la revisión de la mayoría de los supuestos en la inspección realizada, con excepción de la normalidad de los datos. Por lo tanto, a fin de mejorar la distribución de los mismos, se llevó a cabo una transformación logarítmica que minimizó los índices de asimetría y curtosis a valores aproximados entre -1.0 y 1.0, según lo recomienda Huck (2008).

\section{Cuadro 1. Aplicabilidad del modelo de regresiòn}

SUPUESTO

Homocedasticidad

Linealidad

No colinealidad

Normalidad
DESCRIPCIÓN DEL ANÁLISIS

De acuerdo con las pruebas estadísticas de Levene, la muestra reflejó homogeneidad en la varianza, arrojando resultados estadísticamente no significativos

Por medio de una inspección visual de las gráficas de dispersión de puntos, se constataron características predominantes de asociación lineal entre las variables

Los niveles de tolerancia y sus inversos y los factores de inflación de la varianza (FIV) mostraron una baja correlación entre las variables independientes (menores a $20 \%$ ), evidenciando la ausencia de colinealidad

Por medio de la inspección visual de las gráficas de normalidad Q-Q, así como de los índices de asimetría y curtosis, se detectó que algunas variables no se aproximaban totalmente a la normalidad, sino que mostraban una distribución con cierto grado de asimetría positiva y leptocúrtica

Fuente: Elaboraciòn propia. 
Las variables cumplieron con las condiciones de pertinencia para someter a prueba las hipótesis de investigación y lograr inferencias estadísticas. El análisis de regresión múltiple se realizó con el software spss 18, empleando la técnica de estimación por pasos para determinar el modelo con las variables estadísticamente relevantes. Hair et al. (2010) indican que este proceso de estimación secuencial permite examinar diferentes variables independientes por medio de la adición o eliminación selectiva de acuerdo con la magnitud de su contribución a la ecuación de regresión (figura 1).

Tras la estimación de las relaciones propuestas en las hipótesis fue necesario efectuar un análisis de los residuos del modelo de regresión, cuya independencia fue constatada por medio del estadístico Durbin-Watson, el cual tuvo un valor entre el rango de 1.5 y 2.5, como lo recomienda Pardo Merino (2000). Por otro lado, la inspección visual de las gráficas de dispersión de los valores de pronósticos y residuos tipificados mostró varianzas homogéneas, mientras que en la gráfica de regresión parcial la nube de puntos reflejó un patrón de tipo lineal. El histograma de residuos tipificados y la gráfica de probabilidad normal de los residuos evidenciaron que la distribución se aproxima al modelo de probabilidad normal. Además, nuevamente los índices de tolerancia altos y los Fiv bajos demostraron la ausencia de colinealidad en el modelo de regresión.

\section{Resultados}

El modelo de efectos directos propuesto arrojó un resultado estadísticamente significativo $(\mathrm{F}=14.55, \mathrm{p}<.001)$, permitiendo la explicación de poco más de $10 \%$ en la variable dependiente $\left(R^{2}=.109\right)$. Los efectos principales fueron corroborados por dos de las tres pruebas (cuadro 2). En concreto, los efectos principales del tamaño de la empresa $\left(\mathrm{H}_{1}\right)$ y uso de tecnología $\left(\mathrm{H}_{2}\right)$ resultaron estadísticamente significativos para el modelo examinado.

De esta forma, se evidencia que el tamaño de la empresa influye positivamente en su rendimiento $(t=2.07, \mathrm{p}<.05)$, pues por cada unidad de aumento en el número de empleados en la empresa el volumen de ventas en pesos mexicanos tiende a crecer en aproximadamente $13 \%$ al año, considerando que todas las demás variables permanecen constantes. 
Cuadro 2. Resultados del análisis de regresión para los efectos principales

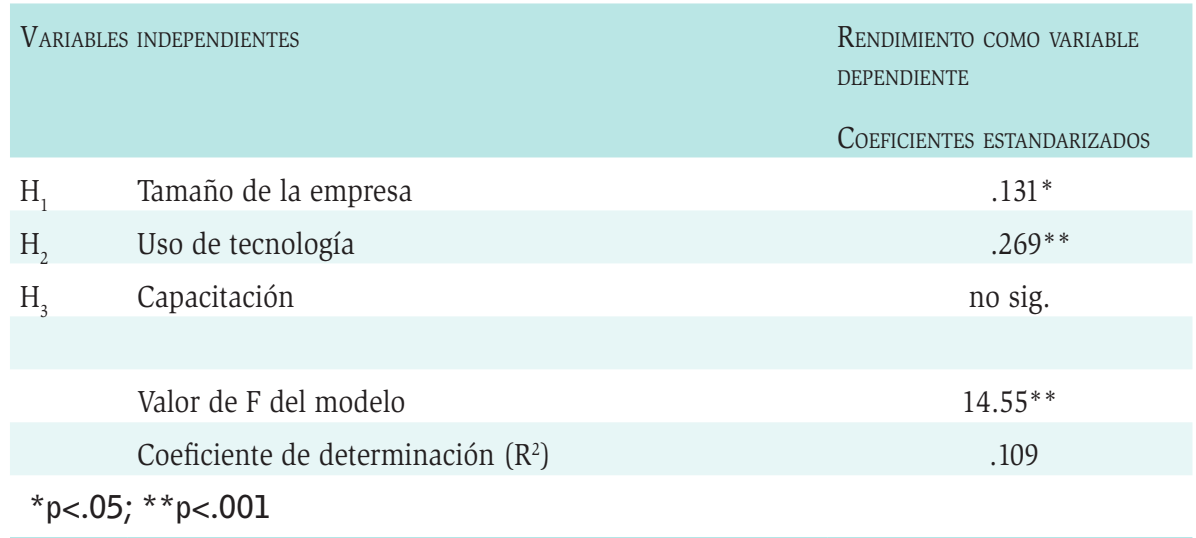

Fuente: Elaboraciòn propia.

También se encontró que el nivel de uso de tecnología en la empresa afecta positivamente su rendimiento $(\mathrm{t}=4.23, \mathrm{p}<.001)$. En otras palabras, por cada empleado que utiliza computadoras para las actividades del negocio, el volumen de ventas en pesos mexicanos tiende a aumentar $27 \%$ anual, considerando las demás variables constantes. Empero, el análisis de regresión indicó que el efecto principal de la capacitación y formación de los empleados $\left(\mathrm{H}_{3}\right)$ no fue estadísticamente significativo en el modelo, lo cual significa que esta variable independiente no tuvo influencia en el rendimiento de las pymes, como se había planteado en la hipótesis de investigación correspondiente.

Con el propósito de examinar los posibles efectos de interacción, al análisis del modelo con los tres efectos principales se le agregaron los cinco términos de interacción preestablecidos. Sin embargo, debido a que las transformaciones logarítmicas pueden alterar la significancia estadística de las pruebas de interacción (Bobko y Russell, 1990), para el análisis de las hipótesis de interacción se emplearon datos sin transformación. Esto no representa problema para la validez de los resultados, ya que en los análisis que emplean muestras de más de 200 casos, así como en modelos con baja complejidad, como el presentado en este trabajo, el posible impacto de una distribución con desviaciones de la normalidad tiende a reducirse (Hair et al., 2010). 
La estimación de esta versión del modelo arrojó un resultado estadísticamente significativo $(\mathrm{F}=4.95, \mathrm{p}<.001)$, con una explicación de casi $15 \%$ en la variable dependiente $\left(\mathrm{R}^{2}=.145\right)$, lo que permite concluir que sí se manifestaron los efectos de interacción planteados (Hair et al., 2010), los cuales fueron corroborados en dos de las cinco pruebas (cuadro 3). Los efectos de interacción entre tipo de servicio y tamaño de la empresa $\left(\mathrm{H}_{6}\right)$, así como entre tipo de servicio y capacitación $\left(\mathrm{H}_{8}\right)$, resultaron estadísticamente significativos en el modelo. Tales interacciones se ilustran en las gráficas 1 y 2 , respectivamente.

\section{Cuadro 3. Resultados del análisis de regresión para los efectos de interacción}

\begin{tabular}{|c|c|c|}
\hline \multicolumn{2}{|c|}{ VARIABLES INDEPENDIENTES } & \multirow{2}{*}{$\begin{array}{l}\text { RENDIMIENTO COMO VARIABLE } \\
\text { DEPENDIENTE } \\
\text { COEFICIENTES ESTANDARIZADOS }\end{array}$} \\
\hline & & \\
\hline $\mathrm{H}_{4}$ & Años en funcionamiento x Tamaño de la empresa & no sig. \\
\hline $\mathrm{H}_{5}$ & Años en funcionamiento x Uso de tecnología & no sig. \\
\hline $\mathrm{H}_{6}$ & Tipo de servicio x Tamaño de la empresa & $-.196^{*}$ \\
\hline $\mathrm{H}_{7}$ & Tipo de servicio x Uso de tecnología & no sig. \\
\hline $\mathrm{H}_{8}$ & Tipo de servicio x Capacitación & $.218^{* *}$ \\
\hline & Valor de F del modelo & $4.95^{* *}$ \\
\hline & Coeficiente de determinación $\left(\mathrm{R}^{2}\right)$ & .145 \\
\hline
\end{tabular}

Fuente: Elaboraciòn propia.

Para facilitar su evaluación en las interacciones, la variable métrica tamaño de la empresa (basada en el número de personal) fue convertida a una variable categórica con tres grupos: micros, pequeñas y medianas empresas. Así, las gráficas del perfil permiten realizar una mejor interpretación de las dos hipótesis corroboradas, en mayor o menor medida, con base en los resultados empíricos. Como se muestra en la gráfica 1, el tipo de servicio (turístico vs. no turístico) y el tamaño de la empresa interactúan positiva y significativamente, pero de modo más marcado respecto al nivel de ventas de empresas que no corresponden a actividades turísticas. Por consiguiente, la interacción planteada $\left(\mathrm{H}_{6}\right)$ se 
corrobora parcialmente, ya que aunque esta impacta en el desempeño de las pymes, el efecto no se refleja principalmente en las de servicios turísticos, como se esperaba.

En lo que se refiere a las micro y pequeñas empresas, no parecen existir grandes diferencias entre servicios turísticos o no turísticos, pero para las de tamaño mediano las diferencias entre los dos tipos de servicio sí parecen más evidentes. De hecho, los resultados indican que el efecto de interacción es muy importante sobre todo en aquellas empresas no turísticas de mayor tamaño (medianas), pero no tanto en las turísticas. Por lo tanto, es necesario tener en cuenta que esta interacción tiene implicaciones en el efecto principal correspondiente a $\mathrm{H}_{1}$, ya que la influencia del tamaño de la empresa en el rendimiento depende del tipo de servicio prestado.

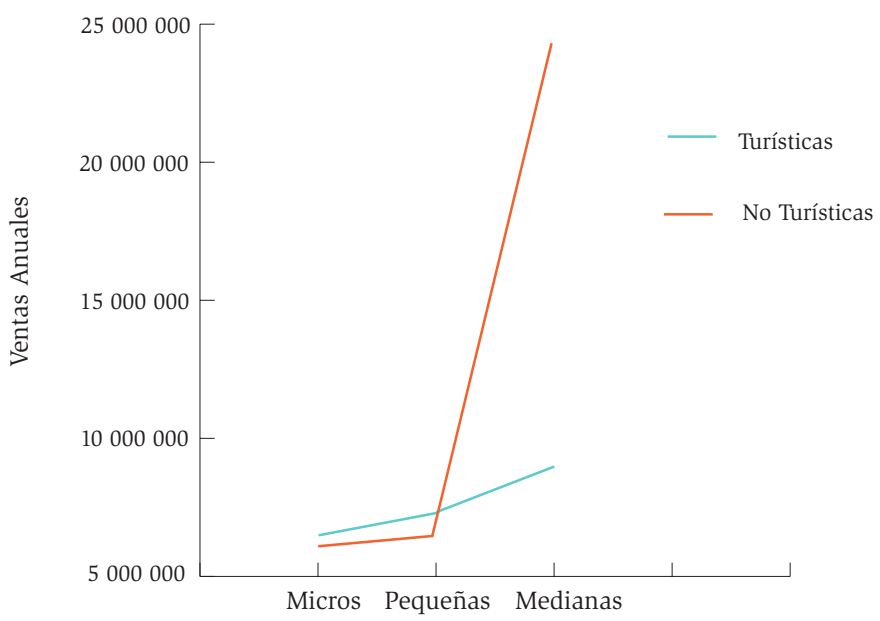

Fuente: Elaboración propia.

Gráfica 1. Perfil de interacción: Tipo de servicio x Tamaño de la empresa 
En la gráfica 2 se puede apreciar un efecto de la interacción entre el tipo de servicio que brinda la empresa y la capacitación de personal que realiza. El análisis evidencia que la capacitación puede tener un impacto mayor en el desempeño de las pymes cuando estas prestan servicios turísticos, pero muy ligero cuando ofrecen servicios no turísticos. Por lo tanto, a diferencia de la interacción anterior, la hipótesis planteada $\left(\mathrm{H}_{8}\right)$ se corrobora de manera completa, ya que además de identificarse un impacto de la capacitación en el desempeño de las empresas, este se muestra más marcadamente en las de servicios turísticos y menos en las de servicios no turísticos. Esto podría tener implicaciones en la relación entre el nivel de capacitación y el rendimiento de la empresa que no fue corroborada previamente $\left(\mathrm{H}_{3}\right)$, aunque debe interpretarse con cautela, ya que las líneas de la gráfica de perfil no alcanzan a cruzarse y, por ende, señalan una interacción ligera.

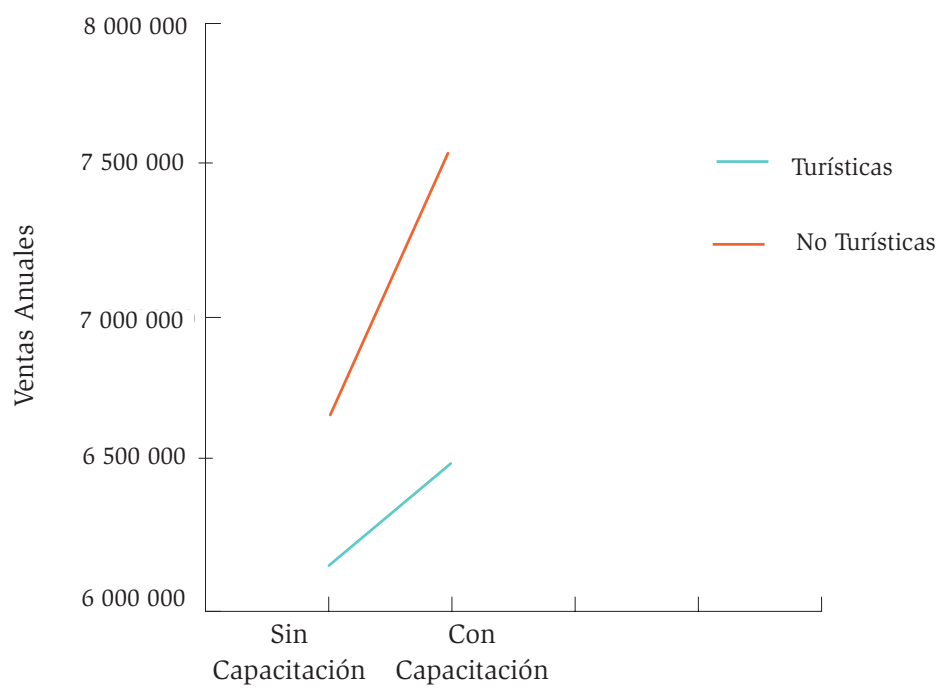

Fuente: Elaboración propia.

Gráfica 2. Perfil de interacción: Tipo de servicio x capacitación 
En cuanto a las demás interacciones esperadas en las hipótesis, el análisis de regresión indicó que ni los años de operación y el tamaño de la empresa $\left(\mathrm{H}_{4}\right)$, ni los años en funcionamiento y el uso de tecnología $\left(\mathrm{H}_{5}\right)$, ni el tipo de servicio y el uso de tecnología $\left(\mathrm{H}_{7}\right)$ fueron estadísticamente significativos en el modelo. Conforme a los resultados, no se comprueba la idea convencional de que un mayor número de años en operación conlleva un crecimiento para la empresa, derivado de un éxito consistente por las actividades de la misma. Tampoco se respalda la noción de que las empresas con más años en funcionamiento tienden a utilizar más tecnologías, ni que las pymes turísticas las usen en mayor grado que las no turísticas.

\section{Conclusiones}

El marco teórico identificó factores como tamaño de la empresa, intensidad en el uso de tecnología, capacitación, años en el negocio y tipo de servicio prestado como posibles antecedentes del rendimiento en las pymes. Las variables independientes fueron examinadas de forma directa e individual, o bien con algunas interacciones entre sí, como predictivas del desempeño de las empresas de servicios en un destino turístico. De acuerdo con lo establecido en las hipótesis, solo las variables independientes tamaño de la firma y uso de tecnología resultaron estadísticamente significativas en los efectos directos de regresión. Además, se lograron identificar dos efectos significativos de interacción entre tipo de servicio y dos de las variables examinadas en los efectos principales o directos.

Respecto al tamaño de la empresa, se puede concluir que, entre más grande sea, hay una tendencia a mostrar un mejor rendimiento medido en términos de ventas anuales, según los datos derivados de las pymes que operan en Cancún. Por lo tanto, incluso en el sector de negocios pequeños existen diferencias en el tamaño y su relación con el volumen de ventas. De acuerdo con Van Dijk (2000), estas diferencias se deben al limitado acceso a los recursos que suelen tener las pequeñas empresas, así como a las características de los apoyos de que disponen.

Sobre los efectos de interacción relacionados con el tamaño de la empresa, los resultados de la investigación no confirmaron la idea de que las pymes de 
mayor tamaño y que llevan más tiempo en el negocio tienen un mejor desempeño en términos de ventas totales. Al menos a partir de los resultados de las pruebas hechas en este contexto, no se puede asegurar que el éxito de las empresas se deba a los efectos conjuntos de su tamaño y el número de años en funcionamiento. Así, estos hallazgos sugieren que una estrategia donde el tamaño de la firma y la magnitud del volumen de operaciones sean centrales puede constituir una ventaja competitiva, independientemente del tiempo que acumule. Por ejemplo, una empresa que incursione en un mercado podría desarrollar este tipo de fortalezas relativamente rápido, sin necesidad de esperar mucho tiempo para conseguir resultados en rendimiento.

Sin embargo, sí se encontró una interacción significativa entre el tamaño de la empresa y el tipo de servicio con las ventas anuales, lo que puede interpretarse como una importante distinción relacionada con el giro de las pymes. En este caso se reveló que el desempeño de los establecimientos no turísticos parece depender en cierta medida del tamaño de sus operaciones, pues las empresas medianas tienden a proyectar mejores resultados que las micro y las pequeñas. Este hallazgo no se reflejó con la misma magnitud en las empresas que prestan servicios de turismo, tal vez porque su éxito comercial depende más de la capacidad de hacer alianzas con las grandes compañías que sirven de canales de distribución, independientemente de su tamaño o de la cantidad de personal que emplean (Torres, 2002).

En cuanto al uso de tecnología, es posible afirmar que a mayor empleo de esta en la empresa hay un mejor desempeño en términos de volumen de ventas. Los resultados sustentan la relevancia que se ha dado en años recientes a fomentar que las pymes incorporen tecnologías de la información en sus operaciones cotidianas, las cuales suelen verse principalmente como herramientas para las grandes firmas. Sin embargo, el análisis de interacciones no confirma que el efecto del uso de tecnología en el desempeño de las pymes en Cancún sea moderado ni por los años en operación ni por el tipo de servicio que brindan. Así, las empresas que busquen ser exitosas en un entorno competitivo deberán considerar cuidadosamente las necesidades de tecnología de su negocio, sin importar el tiempo que tenga en funcionamiento y el tipo de servicio o producto que ofrecen. 
El análisis del modelo también indicó que la capacitación de los empleados tiene una interacción estadísticamente significativa con el tipo de servicio, lo que se refleja en el volumen de ventas anuales. Por lo tanto, al menos para el caso de las pymes turísticas en Cancún, las actividades de capacitación y de formación de personal tienden a ser determinantes en su desempeño en comparación con las empresas de servicios no turísticos, de acuerdo con las relaciones planteadas en las hipótesis. Esto es comprensible si se considera que, al encontrarse en un sector donde el factor humano y la "orientación a las personas” son fundamentales (Goeldner, Ritchie y McIntosh, 2000), las pymes turísticas necesitan implementar actividades de capacitación para mejorar la calidad de sus servicios. Además, demuestra la trascendencia que puede tener la capacitación en los empleos dentro del campo del turismo y la hospitalidad, a pesar de que sean vistos con frecuencia como trabajos que requieren pocas habilidades y destrezas (Baum, 2002).

En resumen, los resultados de la investigación evidencian que las ventas anuales de las pymes en Cancún pueden explicarse en buena medida por el tamaño de la empresa, el uso de tecnología y las interacciones significativas con otras variables independientes. Empero, hay una parte de la variación en el rendimiento de las pymes que no se explica por los constructos considerados en las hipótesis. Esto es consistente con el aserto de Jayawarna, Macpherson y Wilson (2007), quienes enfatizaron otros factores que influyen en el rendimiento de las pequeñas empresas, como las características particulares del producto o servicio vendido, las condiciones del mercado y la economía, etc. Por otro lado, se ha señalado que, debido a que el sector posee un alto grado de heterogeneidad (diferentes tipos de recursos, infraestructura o estilos de administración y liderazgo), su desempeño está sujeto a diversos factores que hacen el estudio de este tipo de negocios algo difícil y complejo (Patton, Marlow y Hannon, 2000).

Con base en los hallazgos presentados, conviene que las futuras investigaciones sobre el tema examinen un mayor número de variables predictivas, así como más variables dependientes que reflejen el desempeño con mayor amplitud. Además, el éxito de las pymes se podría entender mejor al seguir metodologías mixtas, donde el análisis de tipo cuantitativo pueda ser complementado con enfoques de tipo cualitativo. Por último, un diseño de investigación longitudinal podría arrojar resultados más completos para comprender la forma en que los factores inciden en el éxito de las pymes. 


\section{Fuentes consultadas}

APEC (2007). Development of Human Capital for sME Innovation Policies: Turning Technologies into Business Value. Singapur: Asia-Pacific Economic Cooperation Small and Medium Enterprise Working Group.

Baum, T. (2002). "Skills and Training for the Hospitality Sector: A Review of Issues”. Journal of Vocational Education and Training, 54 (3), 343-364. Bobko, P. y C. Russell (1990). "Variance Homogeneity in Interactive Regression: A Clarifying Note about Data Transformations”. Journal of Applied Psychology, 75 (1), 95-96.

Boggio Vázquez, J. y F.J. Vecino Romero (2008). "Emprendimientos e instituciones: El caso Cancún”. Teoría y Praxis, 5, 85-97.

Cestur (2007). Elaboración de un diagnóstico del estado de la investigación turística en el país y generación de un programa de investigación integral para el sector turismo. México: Centro de Estudios Superiores en Turismo.

Cosh, A., J. Duncan y A. Hughes (1998). Investment in Training and Small Firm Growth and Survival: An Empirical Analysis for the UK 1987-95. Londres: Department for Education and Employment (Research Brief, 36).

Dai, W. (2010). "The Impact of Emerging Technologies on Small and Medium Enterprises (SMEs)". Journal of Business Systems, Governance \& Ethics, 4 (4), 53-60.

Ford, A. y D. Brown (2010). "How SMEs are Accessing and Using Enterprise Software: The Experiences of uk Based SMEs from Choice through to Evaluation”, en M. de Castro Neto (ed.), Proceedings of the 4th European Conference on Information Management and Evaluation. Lisboa: Universidade Nova de Lisboa, 86-92.

Goeldner C.R., J.R.B. Ritchie y R.W. McIntosh (2000). Tourism: Principles, Practices, Philosophies. Nueva York: John Wiley \& Sons.

Hair, J.F. et al. (2010). Multivariate Data Analysis. 7a ed. Upper Saddle River: Prentice Hall.

Hernández, P. et al. (2007). "PYMEs, luchar por la sobrevivencia ante el falso discurso oficial”. Caribe Inversión Estudios [en línea]. Disponible en: http://www.caribeinversionestudios.blogspot.mx/2007/05/pymes-luchar-por-la-supervivencia-ante.html [2012, $1^{\circ}$ de mayo]. 
Huck, S.W. (2008). Reading Statistics and Research. 5a ed. Boston: Pearson.

Hunt, S.D. (2010). Marketing Theory: Foundations, Controversy, Strategy, Resource-Advantage Theory. Armonk: M.E. Sharpe.

Hunt, S.D. y R.M. Morgan (1995). "The Comparative Advantage Theory of Competition”. Journal of Marketing, 59 (2), 1-15.

INEGi (2010). Banco de Información Económica [en línea]. Instituto Nacional de Estadística y Geografía. Disponible en: http://www.inegi.org.mx/sistemas/bie/ [2011, 21 de noviembre].

Jayawarna, D., A. Macpherson y A. Wilson (2007). “Training Commitment and Performance in Manufacturing SMEs”. Journal of Small Business and Enterprise Development, 14 (2), 321-338.

Kerlinger, F.N. y H.B. Lee (2000). Foundations of Behavioral Research. 4a ed. Belmont: Cengage Learning.

Kitching J. y R. Blackburn (2002). The Nature of Training and Motivation to Train in Small Firms. Londres: Small Business Research Centre-Kingston University (Research Report RR330).

Massey, C. (2004). "Is the Training Train out of Control? A Case of Evaluation Failure from New Zealand”. Journal of Small Business and Enterprise Development, 11 (4), 458-466.

Matlay, H. (1999). "Vocational Education, Training and Organizational Change: A Small Business Perspective”. Strategic Change, 8 (5), 277-289.

Montazemi, A.R. (2006). "How they Manage IT: SMEs in Canada and the us". Communications of the ACM, 49 (12), 109-112.

Olander, H., P. Hurmelinna-Laukkanen y J. Mahonen (2009). “What's Small Size got to do with it? Protection of Intellectual Assets in SMEs". International Journal of Innovation Management, 13 (3), 349-370.

Pardo Merino, A. (2000). spss Guía para el Análisis de Datos. Madrid: Hispanoportuguesa spss.

Patton, D., S. Marlow y P. Hannon (2000). “The Relationship between Training and Small Firm Performance; Research Frameworks and Lost Quests”. International Small Business Journal, 19 (1), 11-27.

Raymond, L. y J. St-Pierre (2010). "R\&D as a Determinant of Innovation in Manufacturing SMEs: An Attempt at Empirical Clarification”. Technovation, 30 (1), 48-56. 
Secretaría de Economía (2002). Ley para el Desarrollo de la Competitividad de la Micro, Pequeña y Mediana Empresa [en línea]. México: Secretaría de Economía. Disponible en: http://www.siem.gob.mx/portalsiem/ ley_pyme/articulos.asp [2011, 15 de febrero].

Sectur (2007). Programa de apoyo a la competitividad de la micro, pequeña y mediana empresa turística [en línea]. México: Secretaría de Turismo. Disponible en: http://www.sectur.gob.mx/work/sites/sectur/resources/LocalContent/13825/6/Folleto.pdf [2011, 10 de febrero].

------- (2011). El turismo en México [en línea]. México: Secretaría de Turismo. Disponible en: http://www.sectur.gob.mx/work/models/sectur/Resource/1227/1/images/CONCANACO_2011.pdf [2011, 21 de noviembre].

Serrasqueiro, Z. et al. (2010). "Are there Non-Linearities between sme Growth and its Determinants? A Quantile Approach”. Industrial \& Corporate Change, 19 (4), 1071-1108.

Sharma, S., R.M. Duran y O. Gur-Arie (1981). "Identification and Analysis of Moderator Variables”. Journal of Marketing Research, 18 (3), 291-300.

SIEM (2008). Directorio [en línea]. México: Sistema de Información Empresarial Mexicano. Disponible en: http://www.siem.gob.mx/siem/ [2010, 3 de diciembre].

Singh, S.P., R.G. Reynolds y S. Muhammad (2001). "A Gender-Based Performance Analysis of Micro and Small Enterprises in Java, Indonesia”. Journal of Small Business Management, 39 (2), 174-182.

Storey, D. y P. Westhead (2004). "Exploring the Link among Small Firms, between Management Training and Firm Performance: A Comparison between the uK and other oECD Countries". International Journal of Human Resource Management, 15 (1), 112-130.

Torres, R. (2002). “Cancun's Tourism Development from a Fordist Spectrum of Analysis”. Tourist Studies, 2 (1), 87-116.

Van Dijk, M. (2000). “Technological Regimes and Industrial Dynamics: The Evidence from Dutch Manufacturing”. Industrial and Corporate Change, 9 (2), 173-194.

Vargo, S. y R. Lusch (2004). "Evolving to a New Dominant Logic for Marketing”. Journal of Marketing, 68 (1), 1-17. 
Wanhill, S. (2000). "Small and Medium Tourism Enterprises". Annals of Tourism Research, 27 (1), 132-147.

Warner, R.M. (2008). Applied Statistics. From Bivariate Through Multivariate Techniques. Thousand Oaks: SAGE Publications.

Wood, E.H. (2006). "The Internal Predictors of Business Performance in Small Firms. A Logistic Regression Analysis”. Journal of Small Business and Enterprise Development, 13 (3), 441-453.

Yang, C.H. y K.H. Chen (2009). “Are Small Firms Less Efficient?” Small Business Economics, 32 (4), 375-395.

You, J.I. (1995). "Small Firms in Economic Theory”. Cambridge Journal of Economics, 19 (3), 441-462. 
Relación del rendimiento de una empresa, con su tamaño, uso de la tecnología y capacitación

\section{Apéndice}

\section{Reactivos de la encuesta}

Tamaño de la empresa

Por favor indique el número de empleados o colaboradores remunerados que tiene en promedio al año:

$\begin{array}{ll}\text { Valor máximo } & 134 \\ \text { Valor mínimo } & 1 \\ \text { Media } & 12.2\end{array}$

Años de funcionamiento ¿En qué año empezó a operar la empresa?

(En el mismo lugar, aunque con diferentes nombres)

$\begin{array}{ll}\text { Valor máximo } & 30 \\ \text { Valor mínimo } & 1 \\ \text { Media } & 8.2\end{array}$

Uso de tecnología

Por favor proporcione el número de empleados que utilizan computadoras como parte de sus funciones:

$\begin{array}{ll}\text { Valor máximo } & 70 \\ \text { Valor mínimo } & 0 \\ \text { Media } & 5.6\end{array}$

Capacitación en la empresa ¿ ¿La empresa ha llevado a cabo actividades de formación o capacitación de su personal en los últimos dos años?

(Tome en consideración a todos los tipos de empleados)*

$$
\begin{array}{ll}
1=\text { Sí } & 175 \\
0=\text { No } & 67
\end{array}
$$

Tipo de servicio ¿La empresa corresponde a un servicio turístico o de hospitalidad? (Con base en el Sistema de Clasificación Industrial de América del Norte)*

$$
\begin{array}{ll}
1=\text { Sí } & 89 \\
0=\text { No } & 153
\end{array}
$$

Rendimiento Por favor proporcione el volumen de ventas anuales (En pesos, sin incluir los impuestos sobre el valor agregado)

$\begin{array}{ll}\text { Valor máximo } & \$ 40000000 \\ \text { Valor mínimo } & \$ 1200 \\ \text { Media } & \$ 6797421\end{array}$

* Variable categórica dicótoma 
